\title{
First-principles investigations of the magnetocrystalline anisotropy in strained Co-substituted magnetite $\left(\mathrm{CoFe}_{2} \mathrm{O}_{4}\right)^{\text {is }}$
}

\author{
Horng-Tay Jeng ${ }^{\mathrm{a}, *}$, G.Y. Guo ${ }^{\mathrm{b}}$ \\ ${ }^{a}$ Physics Division, National Center for Theoretical Sciences, Hsinchu 300, Taiwan \\ ${ }^{\mathrm{b}}$ Department of Physics, National Taiwan University, Taipei 106, Taiwan
}

\begin{abstract}
The magnetocrystalline anisotropy energy of $\mathrm{CoFe}_{2} \mathrm{O}_{4}$ under uniaxial strain along [ $\left[\begin{array}{ll}0 & 0\end{array}\right]$ direction has been studied by using the linear muffin-tin orbital method within the density functional theory. The calculated uniaxial anisotropy constant $K_{\text {out }}$ is positive for extensive in-plane $\left(\begin{array}{lll}0 & 0 & 1\end{array}\right)$ strains and is negative for compressive in-plane strains. The positive value of $K_{\text {out }}$ indicates that a $\mathrm{CoFe}_{2} \mathrm{O}_{4}$ film under an extensive in-plane strain would show the out-of-plane [0 $\left.0 \begin{array}{ll}0 & 1\end{array}\right]$ magnetization. (C) 2002 Elsevier Science B.V. All rights reserved.
\end{abstract}

Keywords: Ferrite; Magnetic anisotropy energy; Electronic structure

Magnetite $\left(\mathrm{Fe}_{3} \mathrm{O}_{4}\right)$ is an inverse cubic spinel oxide with one iron atom on the tetrahedral $\mathrm{A}$-site and two $\mathrm{Fe}$ atoms on the octahedral B-site per formula unit (fu) above the Verwey transition temperature $(120 \mathrm{~K})$ [1]. The high temperature phase of magnetite is a halfmetallic ferrimagnet with magnetic moment on A-site antiparallel to moment on B-site, resulting in a saturation moment of $4 \mu_{\mathrm{B}} / \mathrm{fu}$. At the Verwey temperature, $\mathrm{Fe}_{3} \mathrm{O}_{4}$ undergoes a metal-insulator transition, accompanied by a structure transition from cubic to monoclinic [2]. The mechanism of the Verwey transition is still a challenging problem to be solved. In the meantime, because of the attractive electronic and magnetic properties as well as the potential industrial applications in magnetic multilayer devices, the magnetism of $\mathrm{Fe}_{3} \mathrm{O}_{4}$ remains to be one of the most interesting areas of research.

In the past decade, investigations of Co substitution were carried out to gain insight into the electronic, magnetic, and anisotropic properties of magnetite. Cobalt ferrite $\left(\mathrm{CoFe}_{2} \mathrm{O}_{4}\right)$ is also a half-metallic material with a smaller saturation magnetic moment of $3.7 \mu_{\mathrm{B}} / \mathrm{fu}$ [3]. $\mathrm{CoFe}_{2} \mathrm{O}_{4}$ crystallizes in the same spinel structure as $\mathrm{Fe}_{3} \mathrm{O}_{4}$ with half of the $\mathrm{B}$-site $\mathrm{Fe}$ atoms replaced by $\mathrm{Co}$

\footnotetext{
${ }^{25}$ Presented at ISPMM/ISAMT2001.

*Corresponding author. Fax: + 886-3-5735081.

E-mail address: jeng@phys.nthu.edu.tw (H.-T. Jeng).
}

atoms (i.e., $\mathrm{Fe}_{\mathrm{A}}[\mathrm{CoFe}]_{\mathrm{B}} \mathrm{O}_{4}$ ), resulting in a lattice constant of $8.39 \AA$ [4]. Mössbauer spectroscopy measurements show that the substitutions distribute randomly over the B-sites throughout the magnetite crystal $[5,6]$. In contrast to the negative fourth order anisotropy constant $K_{1}$ and the [1 111 ] easy axis of cubic $\mathrm{Fe}_{3} \mathrm{O}_{4}$, the $\mathrm{Co}$ atoms in the cubic spinel structure of $\mathrm{CoFe}_{2} \mathrm{O}_{4}$ give rise to a relatively large but positive $K_{1}$ [7] with the [1 00 ] easy axis. The measured Curie temperature slightly decreases with the Co content [8], while impurity effects also decrease the Verwey temperature of doped magnetite [9].

The band structure of cubic $\mathrm{Fe}_{3} \mathrm{O}_{4}$ was calculated within the framework of the local-spin-density approximation (LSDA) by several investigators [10-13]. All these calculations gave a consistent half-metallic result with a magnetic moment of $4 \mu_{\mathrm{B}} / \mathrm{fu}$. The cubic $\mathrm{CoFe}_{2} \mathrm{O}_{4}$ was also demonstrated to be a half-metallic compound with a magnetic moment of $3 \mu_{\mathrm{B}} / \mathrm{fu}$ in band calculations [11]. On the other hand, no magnetocrystalline anisotropy energy (MAE) calculations have been reported perhaps because of the smallness of MAE and the large amount of computing time required. We have recently performed anisotropy energy calculations for cubic as well as strained magnetite [13]. The obtained results agree with the experimental data quite well. The purpose of this work is to investigate the anisotropy energies of the Co-substituted magnetite under uniaxial strains. 
For a cubic crystal bearing a uniaxial strain along [0 01 1], the out-of-plane anisotropy constant is

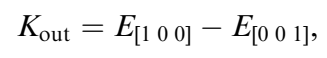

where $E_{[100]}$ and $E_{[001]}$ are the total energies for the magnetization along [1 000$]$ and [0 011$]$, respectively [13]. The computing method used in this work has been described in Ref. [13]. We first performed the selfconsistent scalar-relativistic calculations for each considered strain by using the linear muffin-tin orbitals (LMTO) method. The randomly distributed B-site substitutions of Co atoms were treated by using the virtual crystal approximation. The number of $k$ points over the irreducible wedge (IW) used in the selfconsistent calculations was about 600 over $1 / 16$ of the body-centered tetragonal Brillouin-zone (BZ). After the self-consistent calculation for each strain considered, we used the resultant potential to perform the fully relativistic band structure calculation once for each of the [ $\left.\begin{array}{lll}1 & 0 & 0\end{array}\right]$ and $\left[\begin{array}{lll}0 & 1 & 1\end{array}\right]$ magnetization orientations. The anisotropy energy was calculated as the difference in the eigenvalue sums between the two magnetization directions considered. For the anisotropy calculations, the number of $k$ points over the IW used was approximately 1200 over $2 / 16 \mathrm{BZ}$. The calculated $K_{\text {out }}$ is well converged (within $10^{-4} \mathrm{eV} / \mathrm{fu}$ ) with respect to the number of $k$ points used.

The self-consistent band structures (BS) and density of states (DOS) of the cubic $\mathrm{CoFe}_{2} \mathrm{O}_{4}$ are shown in Figs. 1 and 2, respectively. Due to the same spinel structure as well as the close atomic numbers, the halfmetallic BS and DOS of $\mathrm{CoFe}_{2} \mathrm{O}_{4}$ are quite similar to those of $\mathrm{Fe}_{3} \mathrm{O}_{4}$ [13]. There is a gap of $0.57 \mathrm{eV}$ at the Fermi level for the majority spin, while the conductivity results only from the partially filled minority-spin B-site 3d-band. The calculated spin moment in the $\mathrm{Fe}(\mathrm{A})$ atomic sphere of $\mathrm{CoFe}_{2} \mathrm{O}_{4}$ is $3.39 \mu_{\mathrm{B}}$, being very close to $3.41 \mu_{\mathrm{B}}$ of $\mathrm{Fe}(\mathrm{A})$ in $\mathrm{Fe}_{3} \mathrm{O}_{4}$ [13]. However, the average moment of $2.98 \mu_{\mathrm{B}}$ for B-site of $\mathrm{CoFe}_{2} \mathrm{O}_{4}$ is about $0.5 \mu_{\mathrm{B}}$ smaller than $\mathrm{Fe}(\mathrm{B})$ of $\mathrm{Fe}_{3} \mathrm{O}_{4}$ [13]. This is due to the fact that half of the B-site $\mathrm{Fe}$ atoms are randomly substituted by Co atoms of smaller spin moments. The moment of oxygen is $0.11 \mu_{\mathrm{B}}$ which is the same as that in $\mathrm{Fe}_{3} \mathrm{O}_{4}$ [13]. The total moment of $\mathrm{CoFe}_{2} \mathrm{O}_{4}$ is thus $3 \mu_{\mathrm{B}} / \mathrm{fu}$. Since the spin-orbit coupling is small, the calculated orbital moments affect the spin moments only slightly.

We considered lateral strains in the $\left(\begin{array}{lll}0 & 0 & 1\end{array}\right)$ plane of $\mathrm{CoFe}_{2} \mathrm{O}_{4}$ with the cell volume unchanged. The most significant changes in BS and DOS under strains are in the majority-spin B-site $3 \mathrm{~d}-\mathrm{e}_{\mathrm{g}}$ bands near the Fermi level. This band broadens, especially near the $\Gamma$ point, with increasing magnitude of strains. Thus, at large strains, the top of the B-site 3d-band at the $\Gamma$ point exceeds the Fermi level, and the half-metallic behavior turns into a normal metal state for high compressive $(\varepsilon \leqslant-1.3 \%)$ and high extensive $(\varepsilon \geqslant 2.1 \%)$ strains. In the
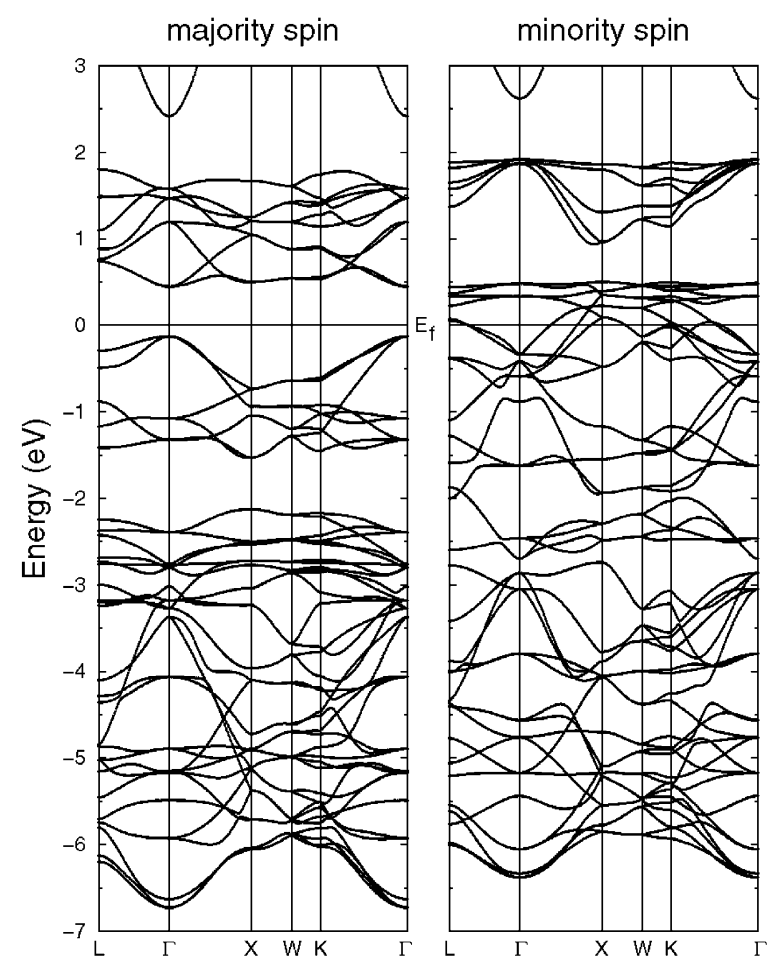

Fig. 1. Band structure of cubic $\mathrm{CoFe}_{2} \mathrm{O}_{4}$.

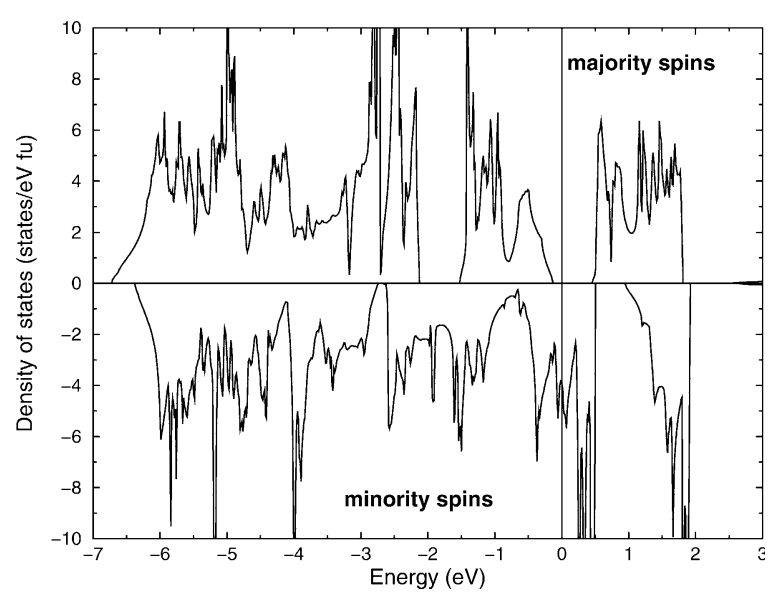

Fig. 2. Spin-decomposed densities of states for cubic $\mathrm{CoFe}_{2} \mathrm{O}_{4}$.

meantime, the total magnetic moment is slightly suppressed during the insulator-metal transition in the majority spin.

The calculated out-of-plane anisotropy constant $K_{\text {out }}$ (circle) of $\mathrm{CoFe}_{2} \mathrm{O}_{4}$ as a function of strain is shown in Fig. 3(a). The corresponding strains for $\mathrm{CoFe}_{2} \mathrm{O}_{4}$ on $\mathrm{NiO}, \mathrm{MgO}$, and $\mathrm{CoO}$ substrates as well as $K_{\text {out }}$ (square) for $\mathrm{Fe}_{3} \mathrm{O}_{4}$ [13] are also shown in the figure. For both 


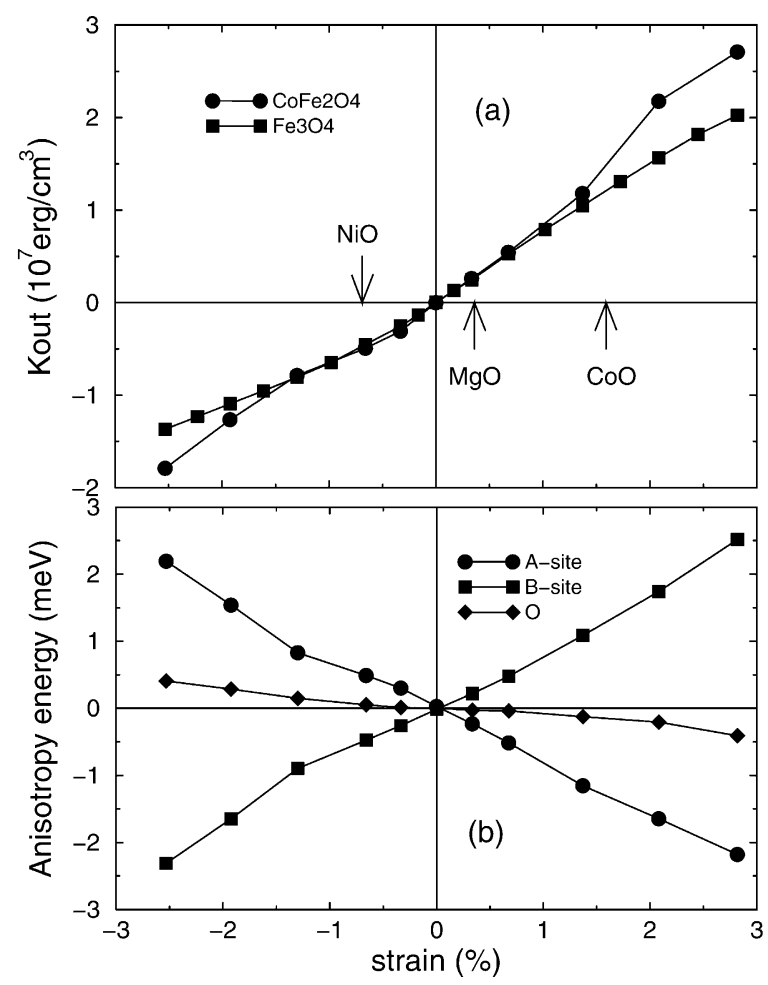

Fig. 3. (a) $K_{\text {out }}$ of $\mathrm{CoFe}_{2} \mathrm{O}_{4}$ and $\mathrm{Fe}_{3} \mathrm{O}_{4}$ as a function of strain. (b) Site-decomposed anisotropy energies for $\mathrm{CoFe}_{2} \mathrm{O}_{4}$ as a function of strain.

$\mathrm{CoFe}_{2} \mathrm{O}_{4}$ and $\mathrm{Fe}_{3} \mathrm{O}_{4}, K_{\text {out }}$ show more or less linear dependences on the strain with slightly larger slopes in the positive strain region. For small strains $(-1.3 \% \leqslant \varepsilon \leqslant 0.7 \%), K_{\text {out }}$ of $\mathrm{CoFe}_{2} \mathrm{O}_{4}$ is about the same as $K_{\text {out }}$ of $\mathrm{Fe}_{3} \mathrm{O}_{4}$. However, for high compressive and high extensive strains, the magnitude of $K_{\text {out }}$ is enlarged by the Co substitutions in the $\mathrm{B}$-site. The negative values of $K_{\text {out }}$ for the negative strains indicate that a $\mathrm{CoFe}_{2} \mathrm{O}_{4}$ film on a substrate with a smaller lattice constant such as $\mathrm{NiO}$ would prefer an in-plane magnetization. On the other hand, for a $\mathrm{CoFe}_{2} \mathrm{O}_{4}$ film on a substrate of larger lattice constant such as $\mathrm{MgO}$ and $\mathrm{CoO}$, the positive $K_{\text {out }}$ implies the out-of-plane magnetization. There have been several measurements on the $K_{\text {out }}$ of the $\mathrm{Fe}_{3} \mathrm{O}_{4}$ film on $\mathrm{MgO}$ and $\mathrm{CoO}$ substrates and remarkably, they agree well with the calculated $K_{\text {out }}$ of $\mathrm{Fe}_{3} \mathrm{O}_{4}$ under extensive strain [13-15]. However, we are not aware of any similar measurement for $\mathrm{CoFe}_{2} \mathrm{O}_{4}$ films.

In Fig. 3(b), we present the site-decomposed anisotropy energies of $\mathrm{CoFe}_{2} \mathrm{O}_{4}$ between [1 000$]$ and [ 00101$]$ directions of magnetization as a function of strain. Despite the rather different electronic structures near the Fermi level for A- and B-sites (Figs. 1 and 2), the contributions from A- and B-sites are about the same amount, but in the opposite sign. The contribution from the oxygen atom is small. Since the number of B-sites is twice as many as that of A-sites, $K_{\text {out }}$ is approximately equal to half of the sum of the anisotropy energies from B-sites.

This work was supported by the National Science Council of the Republic of China (No. NSC89-2119-M007-011, No. NSC90-2112-M002-008).

\section{References}

[1] E.J.W. Verwey, E.L. Heilmann, J. Chem. Phys. 15 (1947) 174.

[2] M. Iizumi, et al., Acta. Crytallogr. 38 (1982) 2121.

[3] J. Smit, H.P. Wijn, Ferrites, Philips, Eindhoven, 1959, p. 149.

[4] R.W.G. Wyckoff, Crystal Structures, Interscience, New York, 1963.

[5] T.L. Templeton, et al., J. Appl. Phys. 73 (1993) 6728.

[6] M. Sorescu, et al., Appl. Phys. Lett. 72 (1998) 2047.

[7] Landolt-Börnstein, New Series III/4b, Springer, New York, 1970.

[8] R.M. Persoons, et al., Phys. Rev. B 47 (1993) 5894.

[9] V.A.M. Brabers, et al., Phys. Rev. B 58 (1998) 14163.

[10] A. Yanase, K. Siratori, J. Phys. Soc. Jpn. 53 (1984) 312.

[11] M. Pénicaud, et al., J. Magn. Magn. Mater. 103 (1992) 212.

[12] Z. Zhang, S. Satpathy, Phys. Rev. B 44 (1991) 13319.

[13] H.T. Jeng, G.Y. Guo, Phys. Rev. B (2001), submitted for publication.

[14] D.T. Margulies, et al., Phys. Rev. B 53 (1996) 9175.

[15] F. C. Voogt, et al., Phys. Rev. B 60 (1999) 11193. 